\title{
J-15 CW Transmission Data Analysis and Comparison with Simulation Results
}

\author{
Yung-Sheng Chiu, Yan-Shiun Chen, Ying-Tsong Lin, and Chi-Fang Chen \\ Department of Engineering Science and Ocean Engineering \\ National Taiwan University
}

\begin{abstract}
Data analysis and 3-D numerical simulations of the acoustic propagation in the ASIAEX SCS experiment are presented in this paper. During the experiment, personnel aboard the Taiwanese ocean research vessel, $O R 3$, periodically deployed and towed the J-15-3 acoustic source to stady the sound propagation in the ocean. One of the emitted waveforms was continuous wave (CW), which had 5 simultaneous $140,260,340,452$ and $560 \mathrm{~Hz}$. Transmission losses of $\mathrm{CW}$ tones are obtained. As for numerical simulations, the wide-angle version of FOR3D is used to calculate transmission losses with background and measured sound speed profile containing intemal tides. The model scenario includes also the sediment and 3-D bathymetry. Results of data analysis and numerical simulations show that TL variations in the data are due to the variations in the sediment and water columns. [Work supported by National Science Council of Taiwan]
\end{abstract}

\section{Introduction}

\section{A. ASIAEX SCS Experiment}

The Asian Seas International Experiment (ASIAEX), South China Sea Component, was a very successful scientific collaboration between the United States, Taiwan and Singapore. The main experiment was conducted in April-May, 2001. ASIAEX-SCS was a volume interaction experiment in the South China Sea. There were also extremely strong geology, geophysics and physical oceanography component to the experiments. The J-15-3 source was a towed acoustic source which emitted a variety of waveforms in the $50-600 \mathrm{~Hz}$ range during six separate tow nuns. Its transmitted frequencies which were complementary to those moored sources, but were selected to prevent interference ocauring.

\section{B. J-15, OR3 Track and Bathymetry}

NRL personnel aboard the R/V OR3 periodically deployed and towed a J-15-3 broadband acoustic sounce. The two main waveforms used were : CW tones and LFM sweeps.

The LFM sweeps also occupied the bandwidth in between the moored source band, going form $50-600 \mathrm{~Hz}$ The CW transmissions were of 5 simultaneous tones : $140,260,340$, 452 , and $560 \mathrm{~Hz}$ which were chosen to hold the band of interest and also not overlap the bands of the moored sources. Fig.1 shows the geographic location inchuding bathymetry contour of the experiment, the deployment of moored acoustic instruments and the receiver array (VLA-HLA). It also shows the tracks of J-15-3 including six separate tow runs along $\mathrm{L}$-shaped tracks paralleled to the paths between mooned sources and receiver arrange.

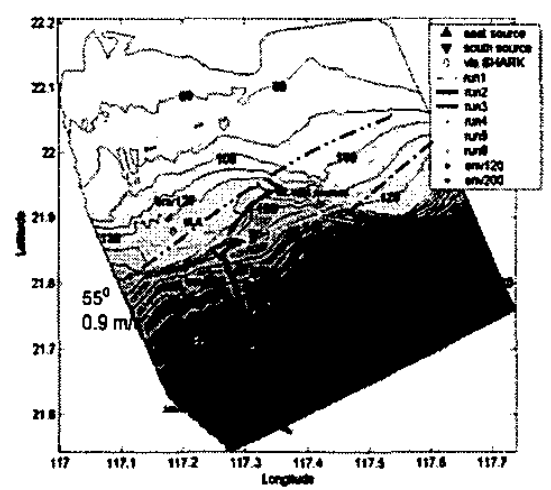

Fig. 1 The geographic bation inchuting bathymetry contour of the experiment and the tracks of J-15-3 including six separate tow nuns

We present the $\mathrm{CW}$ tones transmitted during a segment of J-15-3 RUN2 (which starts at 05/05 23:31, and ends in 05/06 00:23) when internal wave packets were about to pass through the 
VLA. Fig.2 shows the detail bathymetry of the nun of interest. The black asterisks represent the track of the run along the shelf break. The track of interest is nearty along the $200-\mathrm{m}$ isobaths. The blue lines represent every propagation path from source to receiver.

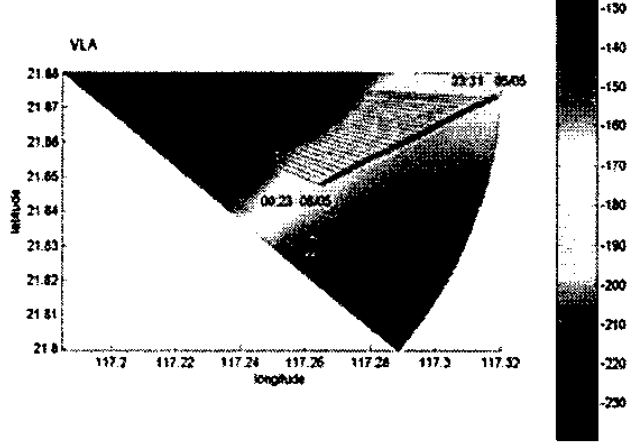

Fig.2 shows the detail bathymetry of the run of interest. The black asterisks represent the track of the run along to the shelf break.

During the period of experiment, the dominant oceanic features are present such as nonlinear internal wave packets, intemal tides or bores were observed at this experimentation area.

\section{Water column interaction / temponal sound speed profile}

Fig. 3 is the standard processing RADARSAT SAR image collected close to the ASIAEX area at 22:14, May 5, 2001, which has some internal wave packets showing as white curve lines. Due to the bathymetry, the propagation direction and the phase velocity of the intemal wave have gradually changed.

In this period, the intemal wave's direction changes from $10^{\circ}$ to $80^{\circ}$ according to $\operatorname{Lin}[1]$. In addition, the phase velocity has decelerated from $1.8 \mathrm{~m} / \mathrm{s}$ to $0.55 \mathrm{~m} / \mathrm{s}$ on May 5 . However, the internal wave field in ASIAEX area sometimes is complicated due to the wave-wave interaction generated by Dongsha Island, which occurred on May 5 and 18[1]. Besides the regular internal wave packet propagating in the westward direction, there is a second wave packet system refracting by Dongsha Island propagating toward the north in ASIAEX area. An interactively twisted wave front therefore is resulted from wave-wave interaction. This wave-wave interaction would cause errors of the internal waves phase speed in this area.
Refer to the SAR image, we clearly see the apparent intemal wave packets coming from the east and propagating in the north-west direction, Moreover, is about to pass through the VLA.

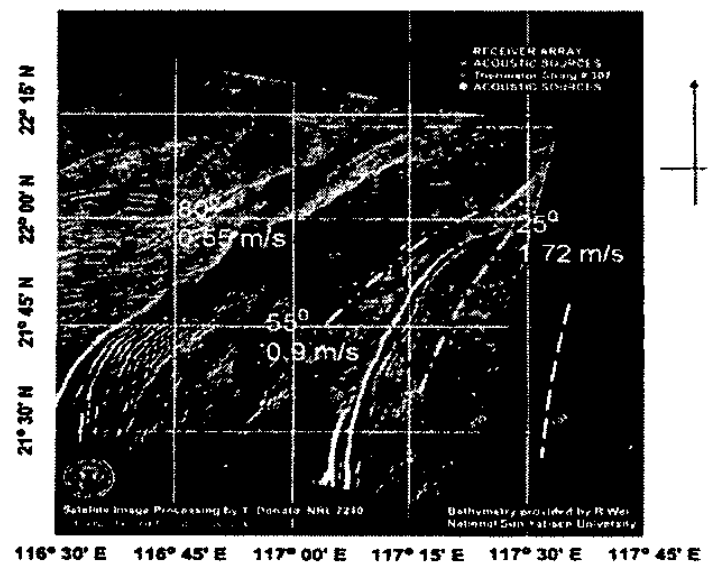

Fig. 3 Due to the influence of bathymetry, the propagation direction and the phase velocity of the intemal wave has gradually changed. The angle between the intemal wavefiont and the North changed from $10^{\circ}$ to $80^{\circ}$. Beside, the phase velocity has decelerated from $1.8 \mathrm{~m} / \mathrm{s}$ to $0.55 \mathrm{~m} / \mathrm{s}$. Satellite Image on ASIAEX-SCS on 05 May 2001 22:14:40. The color lines are hypothesined wave front.

Fig. 4 shows temperature profile in env- 120 and env-200 mooring which is in the vicinity of VLA. Corresponding to the duration of RUN2 of what we are interest in, between 05/05 $23: 31$ to $05 / 0600: 23$, shows the complicated oceanic features is actually occurring and is pretty close to the VLA.

\section{The signal processing for the $\mathrm{CW}$ towed source}

The Fast Fourier Transform with the sliding tapered window is adopted for the frequency analysis of the signal. In order to increase the frequency resolution, the length of the tapered window must be long enough. However, the longer tapered window provides the looser time resolution. Therefore, this opposition has to be balanced. Besides, the Doppler effect of the towed source also needs to be conrected. From navigation records of the $O R 3$, which is a Taiwanese research vehicle and towed the J-15-3 source in the ASLAEX-SCS, the Doppler shift could be estimated. Figure 5 shows that the correction of the Doppler effect is pretty accurate. From this plot, it is obvious to see that the source frequency is shifted because of the Doppler 
Effect and the estimate of the Doppler shift is very agreed with the data.
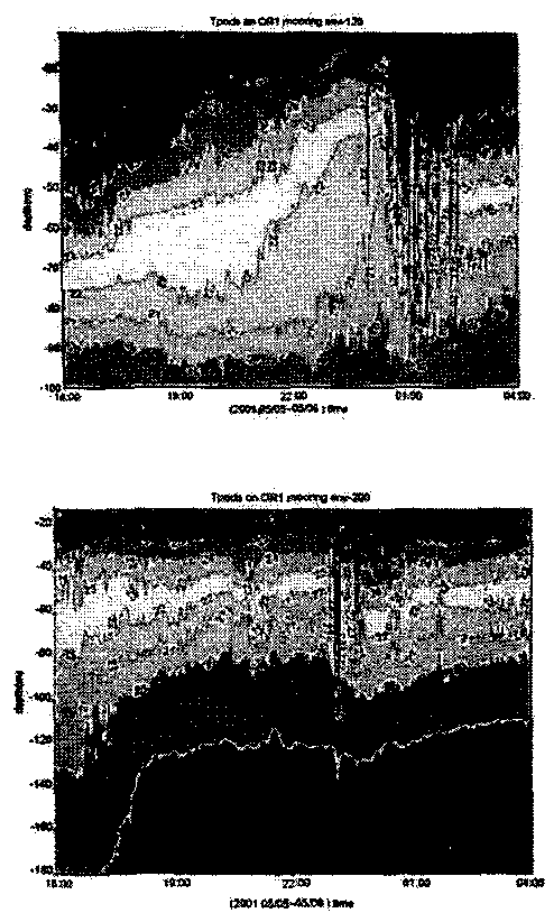

Fig. 4 shows the water column interaction by Tpods on OR1 mooring env- 120 and env- 200 .

After correcting Doppler Effect, the transmission loss could be precisely estimated. Figure 6 shows the result for $140 \mathrm{~Hz}$. The S/N ratio for this frequency was good, and its average is $\sim 10 \mathrm{~dB}$. It is worth to notice that the significant variation of the modal interference pattern is apparent between $10.5 \mathrm{~km}$ and $11.25 \mathrm{~km}$.

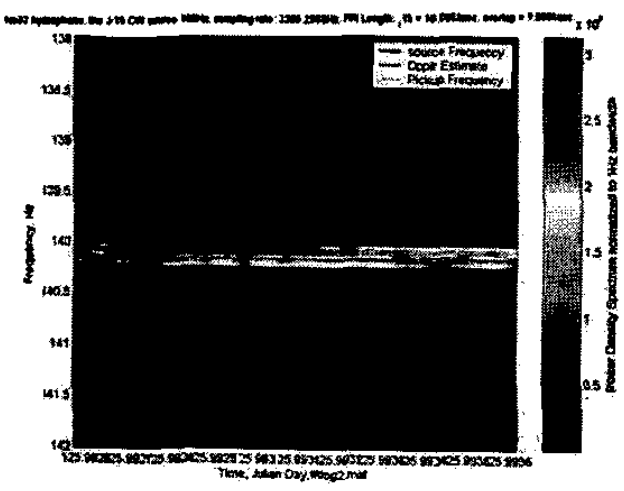

Figure 5: the spectrum gram

\section{TL simulation in the background Model}

As describing as previously, we clearly find that such a simulation scenario is actually complicated to be done. In general, three points seem to be helpful in attermpting to sketch out these anomalous effects on acoustic propagation path.

The first we would like to consider if this anomalous acoustic response is caused by three-dimensional bathymetry effect. Even more important is to considering such oceanic parameters including intemal wave packets and sediment properties.

In studying the effects of intemal waves on acoustic propagation, sediment property is also very necessary to be considered. Recently modeling the effects of shallow water internal wave on acoustic have been published [2]. They not only concern the soliton 'resonance-like' effect on mode coupling [6] but also complement to the interaction of sediment property of the ocean bottom [7]. Just intemal waves and bottom attenuation were both considered then cause the anomalous loss or significantly signal loss. At the resonance frequency, internal waves brings about the modal coupling and a redistribution of energy among the propagating modes. However, if each mode has identical attenuation, the total acoustic field due to the redistributed modes will not appear anomalous signal loss and is almost the same as it was before the redistribution. In general, If the bottom attenuation is not significantly higher for the converted coupled modes, then only a deficient signal loss will occur. For this reason, we need to put to use the gecacoustic model more accurate. Fuller discussion about the geoacoustic parameters will be present in next section.

\section{A. Sediment property and Geoacoustic modeling}

From the chirp-sonar images, not only the bathymetry and the sub-bottom structure are acquired, but also the preliminary estimate of the attemuation coefficient of sound. Using Hamilton's equations[3], other geoacoustic parameters and their profiles are also obtained.

The chirp sonar survey to understand the sub-bottom structure was conducted by Schock[4] in South China Sea area during this experimentation (ASIAEX). The tracks of this 4-day survey 

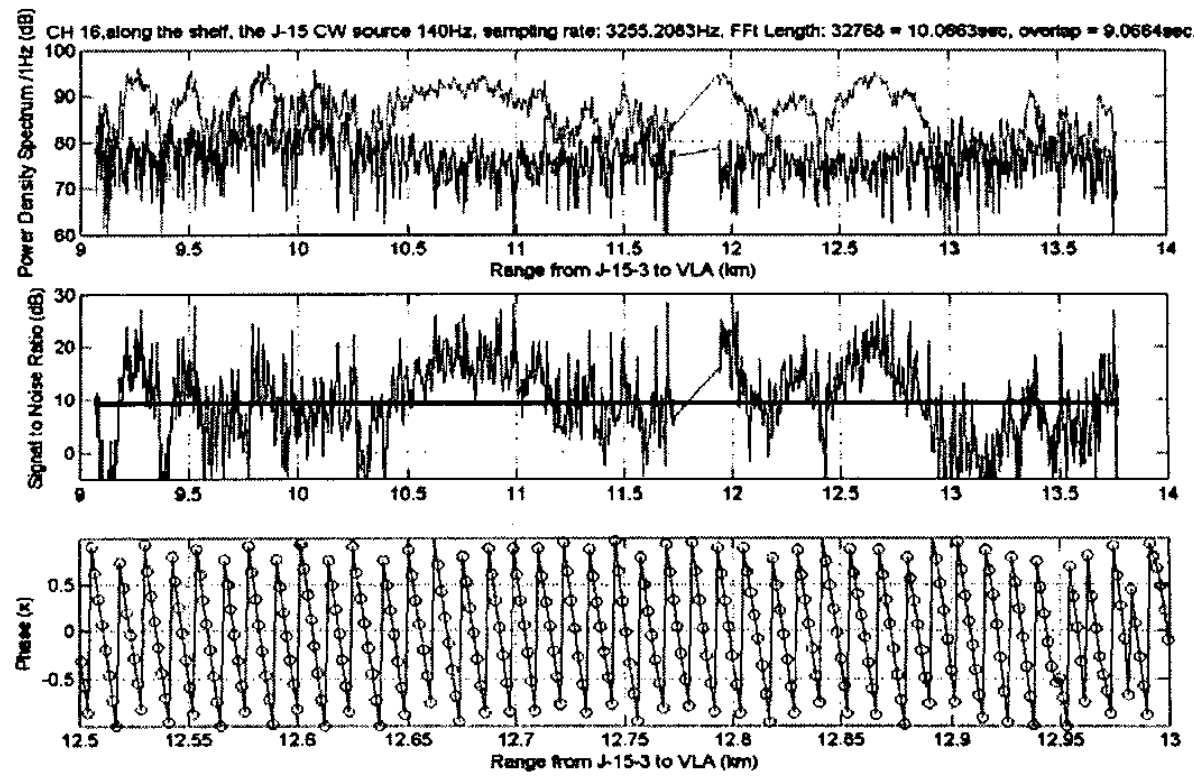

Figure 6: TL analysis of the $140 \mathrm{~Hz} \mathrm{CW}$. The upper panel shows the signal and the noise. The middle shows the SNR and its average, which is 10तB. The lower shows a fraction of the phase of the signal.

are along and across the shelf in this test area. After some analyses, i.e. image processing and the estimation of the attenuation in the sediment, we can get the detailed bathymetries, the simple sub-bottom structure and preliminary geo-acoustic properties of the sediments. It is easy to observe that there is one thin layer covering the basement in the area, both on the shelf and the slope. After analyses of the attenuation coefficient, Steve considers the layer covering the area of the shelf to be silty-sand, and the one covering the slope to be silt.

The chirp sonar survey provides the preliminary estimate of the atternuation properties of the sediment. In Schock's result, there are 26 measured points, 18 points for the track across and 8 along the shelf respectively. From these results, the attemuations are about $0.4 \mathrm{~dB} / \mathrm{m} / \mathrm{kHz}$ on the shelf, $0.15 \mathrm{~dB} / \mathrm{mkHz}$ on the slope and $0.35 \mathrm{~dB} / \mathrm{m} / \mathrm{kHz}$ in the basin.

After obtaining the bathymetry and the bottom attemuation, the bottom sound speed and density environmental model has been done by Lin[5] with Hamilton's formula.

The result gives $1646 \mathrm{~m} / \mathrm{s}$ for silty-sand, $1615 \mathrm{~m} / \mathrm{s}$ for silt and $1749 \mathrm{~m} / \mathrm{s}$ for fine sand. From the sub-bottom structure obtained by the chirp sonar image, they inferred that there was one silty-sand sediment layer on the sand basement in the shelf area, one silt sediment layer on the sand basement in the slope area, and a pure sand layer in the basin area. Fig.7 shows the model of the bathymetry and sub-bottom structure across the shelf

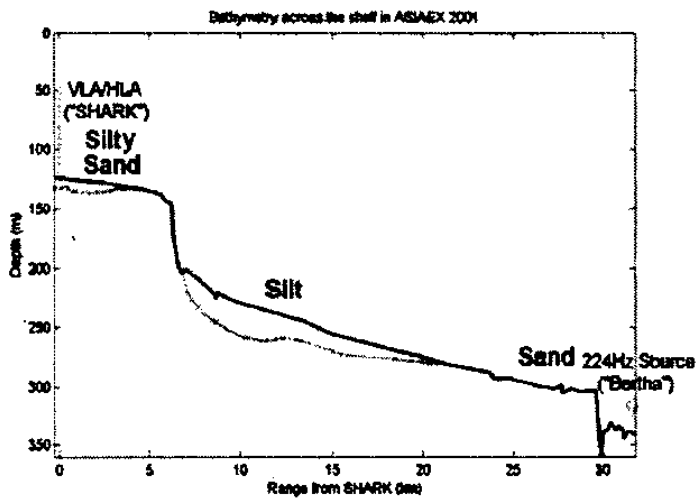

Fig.7 Model of the bathymetry and sub-bottom structure across the shelf

Applying to our simulation scenario, we put his modal in use. Furthermore, we make the assumption that the sediment properties in different depth of every propagation path is almost the same as the acquired parameters from the unique chip sonar 
survey.

\section{B.Background sound speed profile}

For mumerical simulations, the wide-angle version of FOR3D is used to assimilate transmission loss curves with background and measured sound speed profile containing intemal waves. The modal scenario also includes the sediment property and 3D bathymetry. Background sound speed profile is taken from calm sea state in env-200 at the duration May 06, 00:30 to May 06, 04:00 shown in Fig.8.

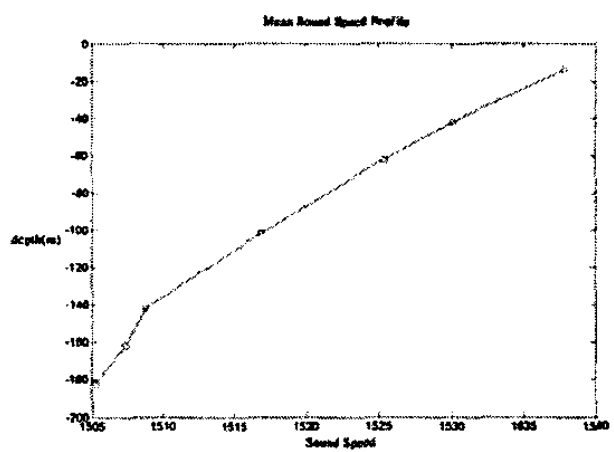

Fig. 8 the background sound speed

\section{C.The background modeling result}

Since the background sound speed profile has been described as previously, the transmission loss was obtained by the wide-angle version of FOR3D to signify the identical acoustic vohme interaction.

$N \times 2 D$ Result - The relative propagation losses versus source bearing in $\mathrm{CH} 3,10,11,12$ of VLA have been shown in Fig.9. The result of background model is in general a tendency toward to the data.

3D Result -Furthermore, Fig.10 shows the 3D simulated result comparing with the $\mathrm{Nx} 2 \mathrm{D}$ result. The background models are actually no apparent differences between 3D simulation scenario and the one of $\mathrm{Nx} 2 \mathrm{D}$.

In this event, we eliminate the effects of 3D bathymetry on acoustic interaction. This result also provides the computation of Nx2D version to be used in the further complicated model scenario is rational and more efficient.

\section{IV.The IW's modeling nesult}

The further modal scenario describes the measured sound speed profile comprehending the internal wave packet and the in-situ oceanic parameters more completely as showing in Fig.11. The upper penal shows the top view of the track of what we are interest in.

The thick blue line indicates the leading internal wave of the packet which is propagating in the west-north direction and the wave front direction maintain $55^{\circ}$. The model scenario of the different propagation path representing the every thin red path of the upper panel shows both water column and bathymetry in the lowe penal. The sound speed profile is taken from the temperature profile in env-120 shown in Fig.4. That is because the total IW's packet has been on the shelf.

As expressing in the two panels, the tuncation of internal wave packet length by the different propagation path is gradually recucing since the propagation path is progressively parallel to the wave front. Similarly, the location of the wave packet is also keeping changing with time. Hence, the more and more shorten wave packet length and the location approaching to the VLA have been expressed in the different propagation path as shown in Fig.11.

Result - Now that the fuller model scenario also including the volume interaction has been done, the simulated transmission loss versus source bearing then be obtain in these four channels. Very interesting to complementary to the result of the background modeling, just source bearing at certain source- range, the anomalous discrepancy will occur. The total transmission losses versus the whole water column among the different modeling framework include both intemal wave packet and background model results has been shown in Fig. 12. The two panels show the pseudocolor plot of the total transmission loss versus source bearing and water column. This clearly shows the apparent different discrepancy occurring as described as previously. 

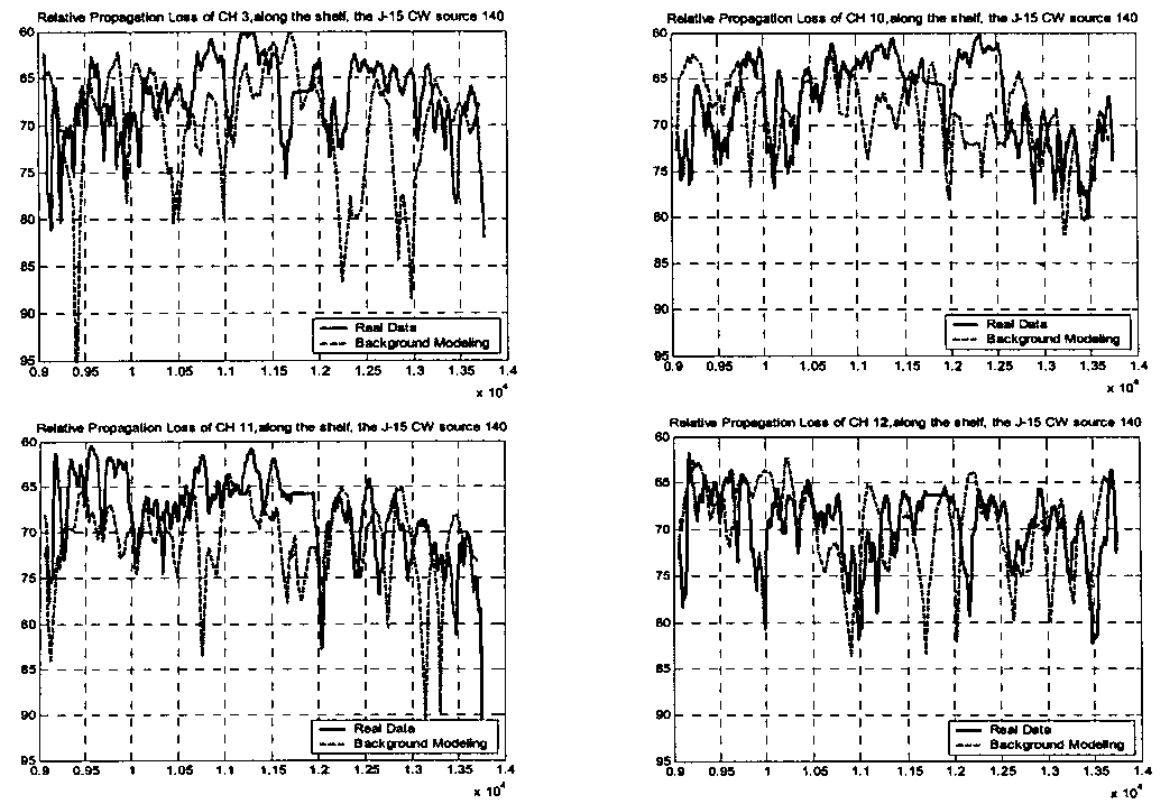

Fig.9 shows the relative propagation losses versus source bearing in $\mathrm{CH} 3,10,11,12$ of VLA. The result of backgnound model is in general a tendency toward to the data.
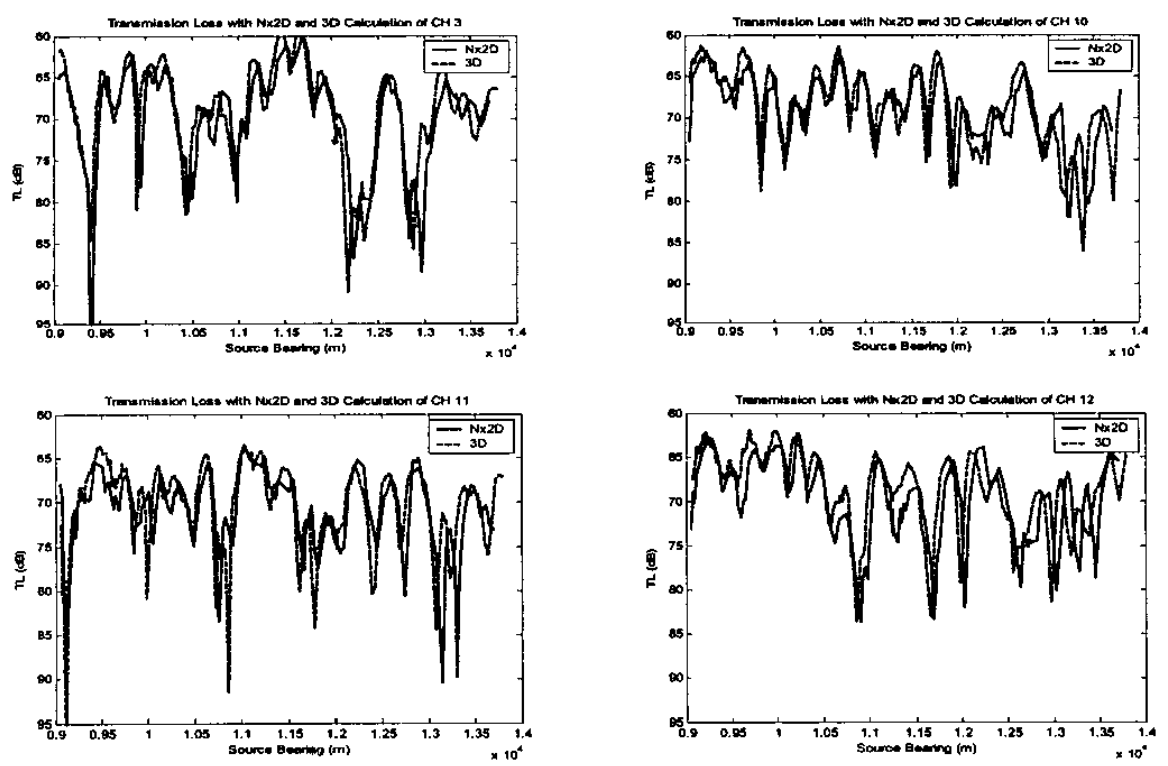

Fig.10 shows the 3D simulated result comparing with the $\mathrm{Nx} 2 \mathrm{D}$ result in $\mathrm{CH} 3,10,11,12$. This result eliminates the effects of 3D bathymetry on acoustic interaction. 

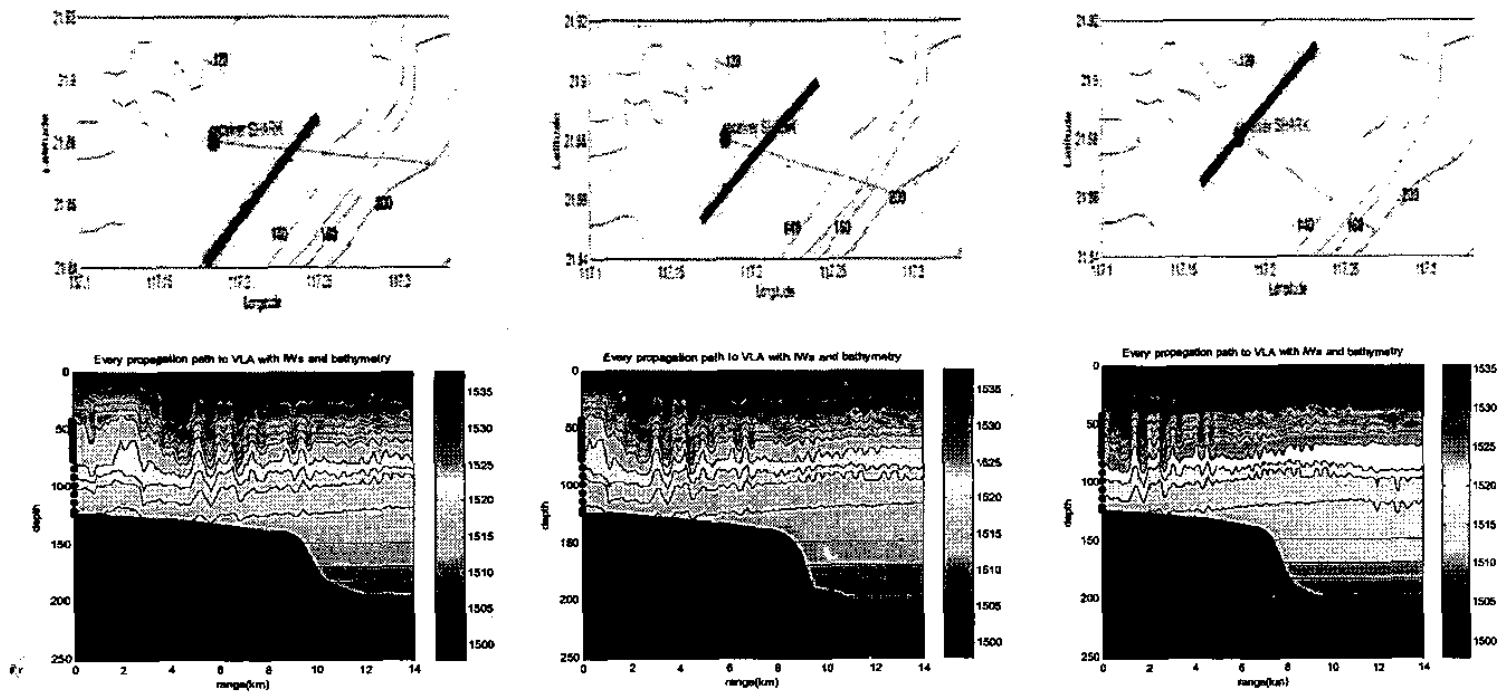

Fig.11 expresses the model scenario including also bathymetry and measured sound speed profile. The upper penal shows the top view of the track. The thick blue line indicates the leading internal wave of the packet which is propagating in the west-north direction and the wave front direction maintain $55^{\circ}$. The lower panel shows the water column and bathymetry corresponding to the red thin line in the upper penal.
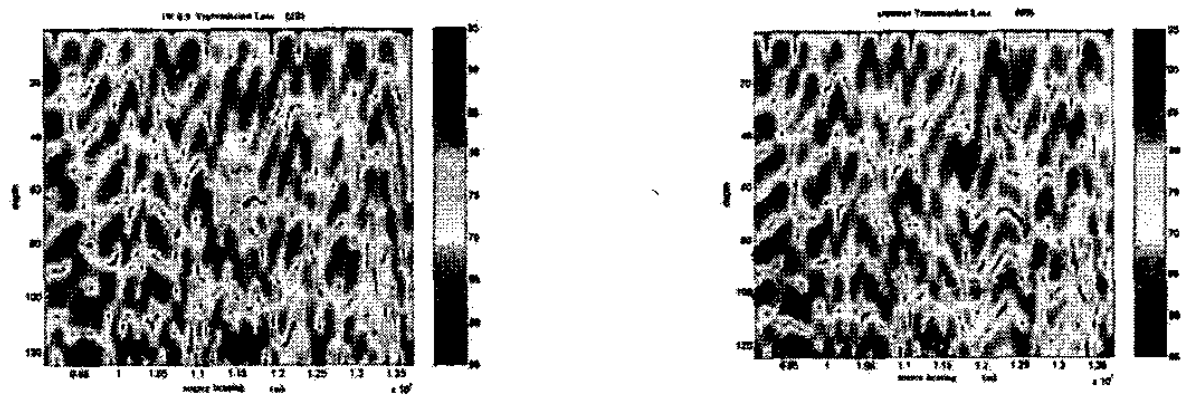

Fig.12 The pseudocolor plots of the total transmission loss versus source bearing and water column. The two panels show the IW's modeling result comparing to the background modeling result. 


\section{Discussion and Conclusion}

In order to match the modeling result to the data, it is necessary to shift the ranges for the data from the analysis of Becker[8], Hawker, Schmidt and Kuperman[9]. That tells the source/receiver motion will impart a shift in the acoustic characteristic, such as TL, horizontal wave numbers of the spectrum and so on. In addition, we perhaps consider another aspect for the comparison with data. That is because the transmission loss curve is too sensitive to identify the moving source acoustic volume interaction. From the result we clearty find that in such real bathymetry is modest 3D effect occurring here. Furthermore, the nonlinear internal wave has evidently caused the acoustic volume interaction in specific source range. The background modeling has been in general a good tendency toward to the data.

\section{Acknowledgement}

The authors would like to thank the ASIAEX team. We especially acknowledge the data collected by ASIAEX team such as RADARSAT SAR images, temporal oceanic data, and so on. This work is supported by National Science Council of Taiwan., ROC, under project.

\section{Reference:}

[1 ] Antony K. Liu*, Yunhe Zhao*, T. Y. Tang**, M. K. Hsu, "Nonlinear Internal Wave Study during ASIAEX", IEEE , 2004

[2] A. C. Warn-Varnas, S. A. Chin-Bing, D. B. King, S. A .Piascek and A. MacNaughton, "Modeling the Effects of Solitons on Acoustics" IOS/WHOI/ONR Internal Solitary Wave Workshop Papers, $6^{\text {th }}$ edition,(1999)

[3]Edwin L. Hamilton, "Geoacoustic modeling of the sea floor," J. Acoust. Soc. Am., Vol 68,

NO.5, 1313-1340 (1980)

[4] S. Schock, "A method for estimating the physical and acoustical properties of the seabed using chirp sonar data," Submitted to IEEE J. Oceanic Eng., Special Issue on the Asian Marginal Seas, 2003.

[5]Ying Tsong Lin," Acoustic Inversions from an Explosive Source in the ASIAEX-SCS Experiment" doctoral dissertation,
Jan.,2004

[6]J. X. Zhou, X. Z. Zhang, and P. H. Rogers, "Resonant interaction of sound wave with internal solitions in the coastal zone," J. Acoust. Soc. Am. 90, 2042-2054 (1991).

[7J. X. Zhou, X. Z. Zhang, and P. H. Rogers, "Effect frequency dependence of sea-bottom attenuation on the optimum frequency for acoustic propagation in shallow water,"J. Acoust. Soc. Am. 82, 287-292 (1987).

[8]K. M. Becker, "Geoacoustic Inversion in Laterally Varying Shallow-Water Environments Using High-Resolution Wavenumber Estimation," DOCTORAL DISSERTATION. February (2002).

[9] K. E. Hawker, A normal mode theory of acoustic Doppler effects in the oceanic waveguide. J. Acoust. Soc. Am., 65(3),

[10]D. Lee and M. H. Schultz, Numerical Ocean Acoustic Propagation in Three Dimensions. Singapore: World Scientific, 1995, pp. 138-144.

[11] A. Tolstoy, "3-D Propagation lssues and Models", $J$. Comput. Acoust., vol. 4, no. 3, pp. 243-271, 1996.

[12]T. F. Duda, and J. C. Preisig, "A Modeling Study of Acoustic Propagation Through Moving Shallow-Water Solitary Wave Packets," IEEE J. Oceanic Engineering, VOL. 24, NO. 1, JANUARY 1999.

[13]C. -S. Chiu, S. R. Ramp, C. W. Miller, J. F. Lynch, T. F. Duda, and T. -Y. Tang, "Acoustic intensity fluctuations induced by South China Sea internal tides and solitions," IEEE J. Oceanic Engineering, 16(5).

[14]D. Tielburger, S. Finette, and S. Wolf, "Acoustic propagation through an internal wave field in a shallow water waveguide" J. Acoust. Soc. Am. 101, 789-808 (1997).

[15]E. L. Hamilton, "Compressional Wave Attenuation in Marine Sediments," Geophysics 37, 620-646 (1972).

[16]E. L. Hamilton, "Sound Attenuation as a Function of Depth in the Sea Floor," J. Acoust. Soc. Am. 59, 528-535 (1976).

[17] Edwin L. Hamilton, "Sound velocity-density relations in sea-floor sediments and rocks," J. Acoust. Soc. Am., Vol. 63 . No.2, 1366-1377(1978) 